\title{
Proline-Directed Androgen Receptor Phosphorylation
}

\section{Yanfei Gao and Shaoyong Chen*}

Division of Hematology/Oncology, Beth Israel Deaconess Medical Center, Harvard Medical School 330 Brookline, MA 02115, USA

\begin{abstract}
The androgen receptor (AR) has been identified for decades and mediates essential steroid functions. Like most of biological molecules, AR functional activities are modulated by post-translational modifications. This review is focused on the reported activities and significance of AR phosphorylation, with particular emphasis on prolinedirected serine/threonine phosphorylation that occurs predominantly on the receptor. The marked enrichment of AR phosphorylation in the most diverse $\mathrm{N}$-terminal domain suggests that targeting AR phosphorylation can be synergistic to antagonizing the C-terminal domain by clinical antiandrogens.
\end{abstract}

Keywords: Androgen receptor; Phosphorylation; Proline-directed serine/threonine phosphorylation; Kinases; Phosphatase

Abbreviations: AF-1/2: AR Activation Function 1/2; AIS: Androgen-Insensitivity Syndrome; AR: Androgen Receptor; CBP: CREB Binding Protein; CHIP: E3 ligase COOH Terminus of Hsp70interacting Protein; Co-IP: Co-immunoprecipitation; CRPC: Castration-resistant Prostate Cancer; DBD: AR DNA-binding Domain; DHT: Dihydrotestosterone; GR: Glucocorticoid Receptor; GRIP1: Glucocorticoid Receptor-Interacting Protein 1; H: AR Hinge Domain; IHC: Immunohistochemistry; JNK: c-Jun N-terminal Kinase; LBD: AR Ligand-Binding Domain; Mdm2: E3 Ligase Murine Double Minute-2; MR: Mineralocorticoid Receptor; NLS: Nuclear Translocation Signal; NTD; AR N-terminal Domain; PCa: Prostate Cancer; PCOS: Polycystic Ovarian Syndrome; PEST Sequence: A Peptide Sequence which is Rich in Proline (P), Glutamic Acid (E), Serine (S), and Threonine (T); Pin1: Peptidyl-Prolyl Cis-Trans Isomerase (PPIase) 1; Poly-Q: Polyglutamine; PP1/2A: Phosphoprotein Phosphatase 1/2A; PR: Progesterone Receptor; SBMA: Spinal And Bulbar Muscular Atrophy; ST: Tumor Antigens Simian Virus 40 Small T Antigen

\section{Introduction}

\section{Androgen receptor (AR)}

The identification of steroid receptors (including AR) in the mid1980 s led to the definition of a family of ligand-mediated transcription factors that occupy specific chromatic locus for gene regulation. The next chapter in advancements is the identification of cofactors participating chromatin remodeling, including histone "writers", "erasers", and "readers" that have continuously been researched up to now. Besides the well-established AR activity in mediating transcriptional activation, recent studies further revealed novel functions of AR in transcriptional repression, genomic translocation, and mTOR activation [1-6]. Abnormal AR activity is associated with various pathogeneses such as male infertility, androgen-insensitivity syndrome (AIS), polycystic ovarian syndrome (PCOS), spinal and bulbar muscular atrophy (SBMA), rheumatoid arthritis, hirsutism, baldness, acne, breast cancer, and prostate cancer (PCa).

The AR molecule is structurally organized into distinct domains: the N-terminal domain (NTD) that has a potent activation function 1 (AF-1), DNA-binding domain (DBD), hinge domain (H), and ligandbinding domain (LBD) that binds to androgens and has a modest activation function 2 (AF-2) (Figure 1A). The AR protein shares highly structural similarities in the DBD and LBD with other steroid nuclear receptor family members, such as glucocorticoids receptor
(GR), mineral corticoid receptor (MR), and progesterone receptor (PR). However, its NTD and hinge regions are unique and structurally disordered, and share marked diversity among family members. The NTD and hinge domain are also less conservative than the DBD and LBD among ARs from different species. Functionally, these distinct domains confer activities such as $\mathrm{N}$-to-C interactions, DNA loading, antiparallel dimerization, and recruitment of cofactors. The AR proteins are also subjected to multiple post-translational modifications such as acetylation, methylation, ubiquitination and sumoylation. This review will be focused on AR phosphorylation, with emphasis on proline (Pro)-directed phosphorylation.

\section{AR phosphorylation}

Theidentification of AR wasimmediately followed by the recognition that the receptor is a phosphoprotein and that phosphorylated AR is localized to the nucleus upon ligand stimulation [7-10]. More extensive studies indicated that AR is synthesized as a single $110 \mathrm{kDa}$ protein that is rapidly converted into a $112 \mathrm{kDa}$ phosphoprotein in the absence of hormone, with constitutive phosphorylation at two Pro-directed serines (Ser650 and Ser94); and that androgens can further induce the expression of a $114 \mathrm{kDa}$ isoform which is phosphorylated at additional residues and associated with AR nuclear activities [11-13]. The distribution of these three isoforms can be attributed to the NTD, in particular the length of the outstanding polyglutamine (poly-Q) stretch and the phosphorylation at two adjacent Pro-directed serines (Ser81 and Ser94) [14].

As shown in table 1 , AR has more than 150 theoretically phosphorylable residues, which are modestly enriched in the hinge and NTD regions. Interestingly, the AR molecular evolution is associated with a decrease in the serines, an increase in the threonines, and a basically unchanged number of tyrosines. Furthermore, the Prodirected Ser (7) and Thr (2) are well conserved among species (Table 1). In addition, the human $A R$ also has abundant glycine $(G, 97)$, proline

*Corresponding author: Shaoyong Chen, Division of Hematology/Oncology, Beth Israel Deaconess Medical Center, Harvard Medical School, 330 Brookline, MA 02115, USA, Tel: 001-617-735-2030; Fax: 001-617-735-2844; E-mail: schen@bidmc.harvard.edu

Received July 30, 2013; Accepted August 23, 2013; Published August 26, 2013

Citation: Gao Y, Chen S (2013) Proline-Directed Androgen Receptor Phosphorylation. J Mol Genet Med 7: 75. doi:10.4172/1747-0862.1000075

Copyright: (c) 2013 Gao Y, et al. This is an open-access article distributed under the terms of the Creative Commons Attribution License, which permits unrestricted use, distribution, and reproduction in any medium, provided the original author and source are credited 


\begin{tabular}{|c|c|c|c|c|c|c|c|c|}
\hline & Total AA & Serine & Theonine & Tyrosine & $\mathrm{S} / \mathrm{T} / \mathrm{Y}$ & Percentage & Pro-Ser & Pro-Thr \\
\hline $\begin{array}{l}\text { Human AR } \\
\text { Chimpanzee AR } \\
\text { Mouse AR } \\
\text { Rat AR }\end{array}$ & $\begin{array}{l}919 \\
911 \\
899 \\
902\end{array}$ & $\begin{array}{l}81 \\
81 \\
87 \\
92\end{array}$ & $\begin{array}{l}37 \\
37 \\
35 \\
33\end{array}$ & $\begin{array}{l}33 \\
33 \\
34 \\
33\end{array}$ & $\begin{array}{l}151 \\
151 \\
156 \\
158\end{array}$ & $\begin{array}{l}\% 16.43 \\
\% 16.58 \\
\% 17.35 \\
\% 17.52\end{array}$ & $\begin{array}{l}7 \\
7 \\
7 \\
7\end{array}$ & $\begin{array}{l}2 \\
2 \\
2 \\
2\end{array}$ \\
\hline $\begin{array}{l}\text { Human AR NTD } \\
\text { Human AR DBD } \\
\text { Human AR Hinge domain } \\
\text { Human AR LBD }\end{array}$ & $\begin{array}{c}537 \\
90 \\
45 \\
247\end{array}$ & $\begin{array}{c}56 \\
4 \\
4 \\
17\end{array}$ & $\begin{array}{c}20 \\
5 \\
6 \\
6\end{array}$ & $\begin{array}{c}20 \\
5 \\
1 \\
7\end{array}$ & $\begin{array}{l}96 \\
14 \\
11 \\
30\end{array}$ & $\begin{array}{l}\% 17.88 \\
\% 15.56 \\
\% 24.44 \\
\% 12.15\end{array}$ & $\begin{array}{l}6 \\
0 \\
1 \\
0\end{array}$ & $\begin{array}{l}1 \\
0 \\
0 \\
1\end{array}$ \\
\hline
\end{tabular}

Table 1: Characterization of AR amino acid composition regarding theoretically phosphorylable residues. GenBank entry: human AR (M20132.1); chimpanzee AR (NM_001009012.1); mouse AR (NM_013476.3); and rat AR (NM_012502.1). AA: amino acid; S: Serine/Ser; T: Threonine/Thr; Y: Tyrosine/Tyr.

(P, 74), and glutamine $(\mathrm{Q}, 69)$. Computational calculation indicates that the Pro-directed Ser residues are subjected to phosphorylation by Pro-directed Ser/Pro kinase (Figure 1B). While the Acid Ser/Pro kinase theoretically covers AR but the C-terminal LBD, the Baso Ser/Pro kinase can potentially phosphorylate all four domains (Figure 1B). This result is in concordance with an analysis by a distinct program, indicating that the N-terminal region (NTD-DBD-H; in particular the NTD) is the major phosphorylation locus of AR (Figure 1C). Consistently, multiple studies indicated that the NTD is the predominant AR phosphorylation region and the phosphorylation occurs mainly on Ser and Thr residues [15-17]. Lower phosphorylation frequency identified in DBD and especially the LBD is rather due to their highly structural conformation than low percentage of phosphorylable residues, considering that phosphorylation occurs mainly on intrinsically unstructured locus (Figure 1C and Table 1) [18].

The enrichment of phosphorylation at NTD implies its function in AR-mediated transactivation. Indeed, AR phosphorylation status is strongly correlated with the transcriptional function and the agonistic activity of ligands [19]. Consistently, the NTD (the major AR phosphorylation region) bears AR activation mediated by the HER2/ EGFR and IL-6/ MAPK pathways [20,21]; and Aurora-A and Ack1 also activate AR by phosphorylating the NTD (Thr282/Ser293 versus Tyr267/ Tyr363, respectively) [22,23]. Furthermore, AR phosphorylation is involved in AR degradation by the proteasome-dependent pathway: while phosphorylation at Ser578 promotes AR-Mdm2 (E3 ligase murine double minute-2) association and AR degradation, phosphorylation on Tyr (534) attenuates AR ubiquitination and interaction with the E3 ligase CHIP (COOH terminus of Hsp70-interacting) protein, leading to increased AR expression [24-26]. In addition, AR phosphorylation is also linked to additional modifications such as AR acetylation [27].

\section{Proline-directed AR phosphorylation}

Despites the scattered reports on AR phosphorylation at various residues, it is well documented that the receptor phosphorylation occurs predominantly on the Pro-directed serines, as evidenced by the studies based on phosphoamino acid and mass-spec analyses (data not shown) [28]. As indicated in figure 1A and table 1, AR totally has seven Pro-directed serines, with six located at the NTD and one at the hinge region. Functionally, AR phosphorylation at Ser308 by cyclin D3/ CDK11p58 reduced transcriptional activity [29], while the functional significance of S515 phosphorylation appears different between exogenous and endogenous studies [13,30,31]. In addition, S424 and S515 phosphorylation contributes to AR nuclear localization and functions against receptor aggregation upon hormone treatment [32].

Next, we will concentrate on three Pro-directed Ser residues (Ser81, Ser94, and Ser650) that are most robustly phosphorylated based on multiple phosphoamino acid and mass-specanalyses (Figure 2) $[12,14]$. Although Ser81 is apparently the highest androgen-stimulated
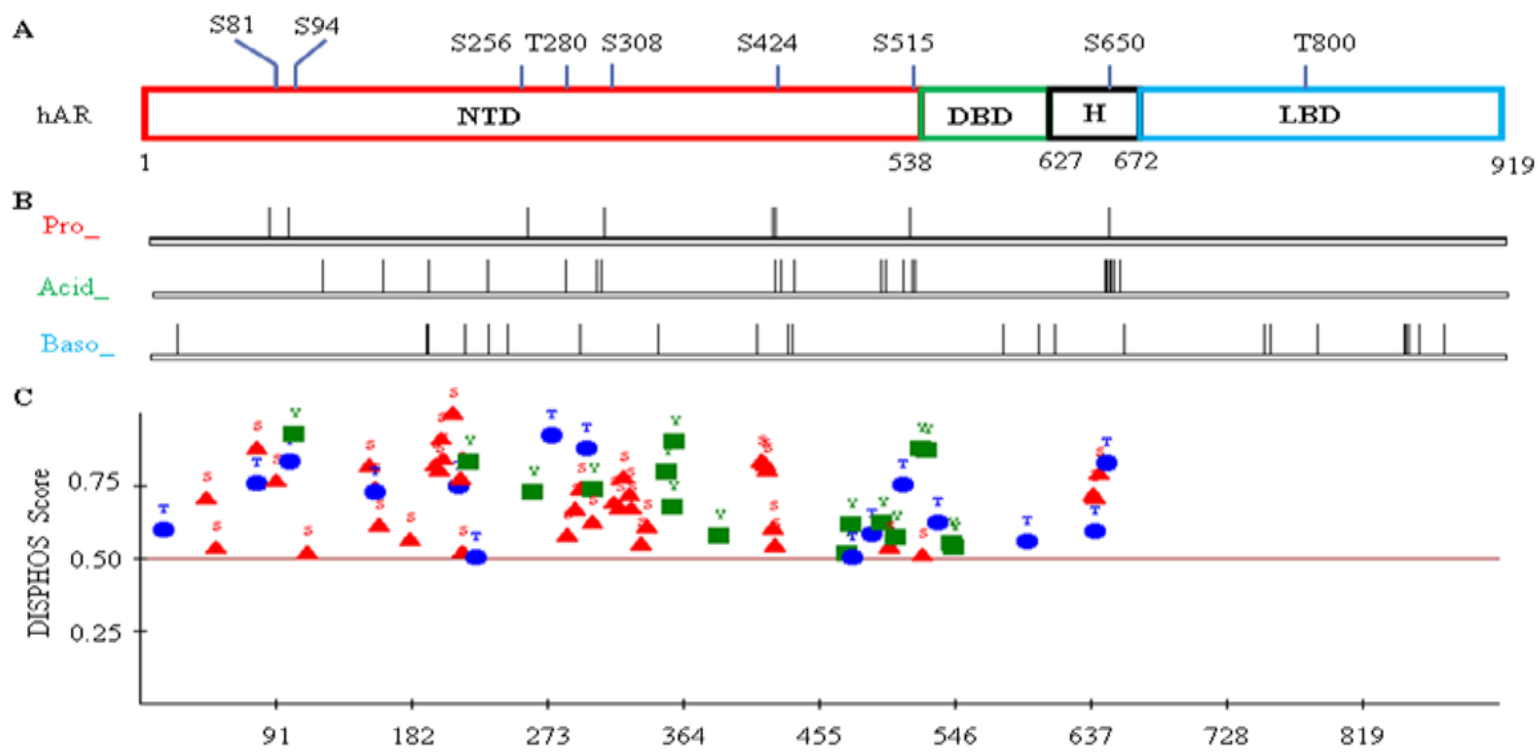

Figure 1: Theoretical and computational characterization of human androgen receptor (hAR) phosphorylation.(A) hAR amino acid linear organization indicated of structural domains and proline(Pro)-directed phosphorylable residues (GenBank: M20132.1); (B) Motif Scan Graphic analysis ofhAR subjected to Pro-directed Ser/ Pro kinase (Pro_ST_Kin or Pro_), Acid Ser/Pro kinase (Acid_ST_Kin or Acid_); and Baso Ser/Pro kinase (Baso_ST_Kin, or Baso_) (http://scansite.mit.edu); (C) Phosphorylation analysis of hAR by the DISorder-enhanced PHOSphorylation predictor (DISPHOS, http://www.ist.temple.edu/DISPHOS) program. 
AR phosphorylation residues, this event cannot be readily captured by mass-spec due to its particular embedment in the Poly-Q stretch that would compromise the fragmentation efficiency during digestion and processing (Figure 2 and 3) [17].

\section{Pro-directed Ser81 phosphorylation}

Ser81 is the most stoichiometrically serine residue phosphorylated in response to androgens and its phosphorylation occurs with distinct dynamics compared to other AR phosphorylation residues [12,17]. The particular activities of Ser81 phosphorylation can be attributed to its extraordinary positioning in an unusual polyglutamine (poly-Q) stretch in the NTD (Figure 3A). Interestingly, a linear increase in the length of poly- $\mathrm{Q}$ is proportional to the time of animal divergence, suggesting an association of polyglutamine expansion with evolution of the higher primate species [33]. Pathologically, the polymorphic poly-Q is causative to certain neurodegenerative diseases, as exemplified by the neuromuscular disorder SBMA [34]. In the molecular settings, expansion of the poly-Q track led to abnormal AR protein folding, aggregation, and interaction with other proteins, resulting in excessive AR degradation and compromised AR transcriptional capacity [35].

In the prostate cancer ( $\mathrm{PCa})$, Ser81 phosphorylation contributes to cell growth, AR-mediated transcription, and AR sensitivity to ligand [3639]. Although transient transfection assay yielded little effect of Ser81 phosphorylation on AR-mediated transcription [17,36], studies based on PCa and endogenous genes indicated that this phosphorylation had pronounced effects on AR nuclear distribution, chromatin binding, and transactivation functions $[37,39,40]$. Consistently, attenuation of AR Ser81 phosphorylation by antagonists for CDK1, CDK9, TOPO1 (topoisomerase I) and HER2 led to decreased PCa cell growth and AR nuclear functions such as chromatin binding and transcriptional activation $[37,39,41,42]$. Interestingly, AR Ser81 phosphorylation can also function in the PCa epithelial-stromal interactions, mediated by the ERK pathway that may directly phosphorylate this residue $[36,43]$.

Mechanistically, the initial work from our Lab identified Cdk1 as a Ser81 kinase that can phosphorylate Ser81 to stimulate AR nuclear functions [36]. Further studies indicated that CDK9 specifically phosphorylate AR at Ser81 upon androgen stimulation, leading to productive AR chromatin binding for sustained transcription [37,39]. Consistently, DNA binding has been implicated in Ser81 phosphorylation and androgens-induced AR localization to the active chromatin may be associated with phosphorylation by specific protein kinase occupying the locus $[14,44,45]$. Together, these findings suggested that CDK1-mediated Ser81 phosphorylation may account for the basal Ser81 phosphorylation that can initiate AR loading to the chromatin locus, followed by CDK9-mediated phosphorylation that is coupled to transcriptional activation (Figure 3B). At molecular levels, Ser81 phosphorylation is implicated in AR nuclear distribution and its interaction with co-factors, such as CBP (CREB binding protein) and GRIP1 (glucocorticoid receptor-interacting protein 1) [45]. In addition, a recent report also indicated that Ser81 phosphorylation

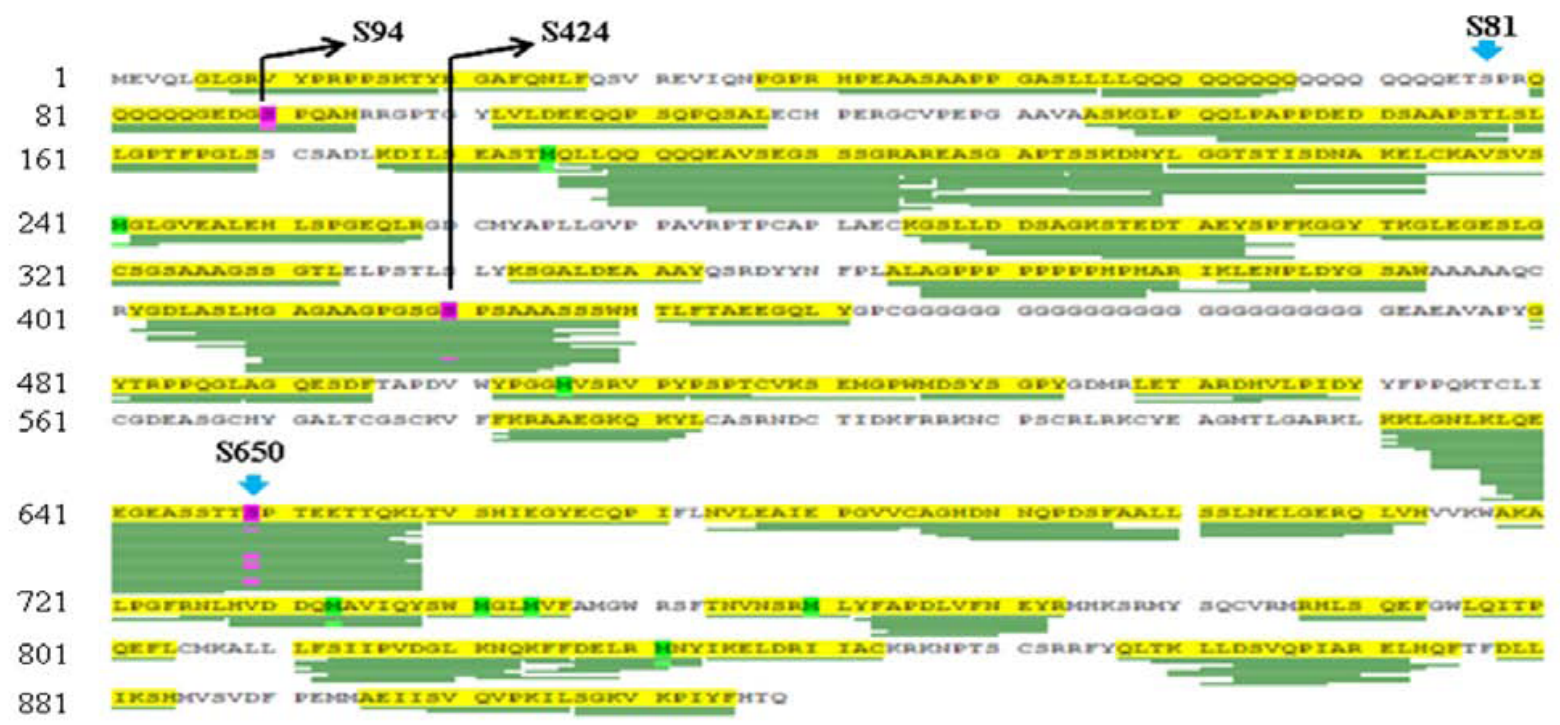

Figure 2: A typical mass-spec analysis in AR study. LNCaP cells in androgen-deprived medium were treated with DHT (dihydrotestosterone) and AR was harvested by Co-IP (Co-immunoprecipitation) for mass-spec analysis that was aligned to human AR (GenBank: M23263.1). Highlighted are identified phosphorylation corresponding to Ser94 and Ser650(GenBank: M20132.1), with Ser424 at lower frequency. Phosphorylation on Ser81 is not identified, likely due to its particular location that affects fragmentation during processing.

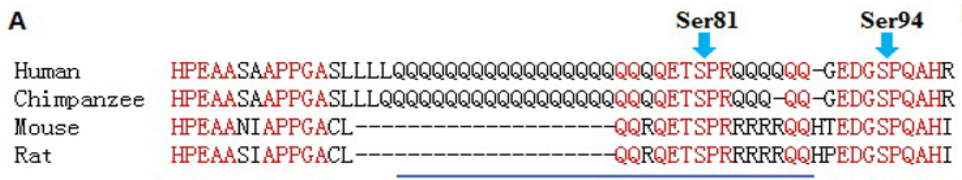

Polyglutamine (Poly-Q)

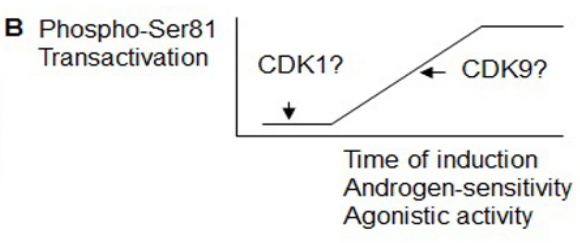

Figure 3: AR linear amino acid sequences in the vicinity of Ser81 and Ser94 residues.(A) Alignment of human, chimpanzee, mouse and rat AR with highlighted conserved residues (in red) and polyglutamine region (underlined); (B) Schematic drawing indicates that Ser81 phosphorylation is correlated to AR functional activities. 
mediates the interaction between AR and Pin1, a peptidyl-prolyl cistrans isomerase (PPIase) that specifically isomerizes Pro-directed phospho-Ser/Thr motifs [46].

\section{Pro-directed Ser94 phosphorylation}

The Ser94 locates to the C-terminal of the NTD poly-Q track (Figure 3A) and its phosphorylation can occur in the absence of ligands [12]. Ser94 together with Ser81 and Ser650 are the three Pro-directed residues that are most substantially phosphorylated AR residues (Figure 2) [17]. The functional significance of Ser94 phosphorylation is unknown; although a bias was found for Ser94 phosphorylated AR distribution in the cytoplasm in the absence of androgens [40]. In addition, increased Poly-Q length is associated with enhanced Ser94 phosphorylation while transient transfection study has indicated that Ser94 phosphorylation has minimal effect on AR-mediated activation of exogenous promoters $[13,17,33]$. The Ser94 kinase(s) are also unknown, although CDK1 and CDK5 but not CDK9 can be the candidates [36,37]. Considering Ser94 phosphorylation can happen in the absence of androgens, it remains to be determined whether this phosphorylation is involved in the interaction between AR with the HSP90 complex in the cytoplasm that binds to and stabilizes the newsynthesized AR proteins [47].

\section{Pro-directed Ser650 phosphorylation}

The Ser650 in the only Pro-directed serine residue that locates outside of the NTD. It resides in the hinge region and centers on the diverse PEST sequence that potentially mediates AR protein degradation (Figure 4). The hinge region contains part of the NLS (nuclear translocation signal) and regulates AR transactivation and nuclear localization, and is one major target site for modifications (acetylation, ubiquitination and methylation) [48,49]. The hinge region is also enriched in phosphorylable residues and indeed, massspec analysis has identified phosphorylation occurring at Ser650 and several adjacent residues (Table 1, Figure 4; data not shown). Although transient transfection assays suggested that 650 phosphorylation has no or minimal effects on AR functional activities, study based on endogenous AR indicated that the stress kinases (JNK (c-Jun N-terminal kinase) and p38) regulate Ser 650 phosphorylation and AR nuclear export $[13,17,28]$. The functions of phosphorylation at the adjacent residues (Ser646, Ser647, and Thr652) are unknown (Figure $4)$.

\section{Phosphoprotein phosphatases targeting the Pro-directed Ser/ Thr residues of AR}

The findings that phosphorylated $\mathrm{AR}$ is transcriptionally active are essentially consistent with the observations that AR dephosphorylation impairs receptor functional nuclear activities like ligand binding [50,51]. Consistently, the tumor antigens simian virus 40 small $t$

\begin{tabular}{ll} 
& \multicolumn{1}{c}{$\begin{array}{c}\text { Ser650 } \\
W \checkmark v\end{array}$} \\
Human & ARKLKKLGNLKLQEEGEASSTTSPTEETTQKLTVSHIEGYECQPI \\
Chimpanzee & ARKLKKLGNLKLQEEGEASSTTSPTEETTQKLTVSHIEGYECQPI \\
Mouse & ARKLKKLGNLKLQEEGENSNAGSPTEDPSQKMTVSHIEGYECQPI \\
Rat & ARKLKKLGNLKLQEGENSSAGSPTEDPSQKMTVSHIEGYECQPI \\
\cline { 2 - 2 } &
\end{tabular}

Figure 4: AR linear amino acid sequences of the hinge domain. Alignment of human, chimpanzee, mouse and rat AR with highlighted conserved residues (in red) and PEST sequences (underlined). Black arrow heads indicated additional phosphorylation residues (Ser646, Ser647, and Thr652) identified in the hinge region by our mass-spec studies (data not shown). antigen (ST) can mediate PP2A (phosphoprotein phosphatase 2A) binding to AR, leading to AR dephosphorylation at five Pro-directed phosphoserines in the NTD and reduction in AR activities [51]. Significantly, PP2A activity is attenuated in the androgen-independent C4-2 PCa cells as compared with the parental androgen-dependent LNCaP cells [52]. Furthermore, PPP2R2C (a PP2A regulatory subunit) was down-regulated in advanced $\mathrm{PCa}$ to drive castration-resistance [53]. In addition, study from our Lab indicated PP1 (phosphoprotein phosphatase 1) stimulates AR nuclear functions (in opposite to that of the PP2A), mediated by PP1-elicited dephosphorylation of Ser650 in the hinge region [54]. These findings are fundamentally in line with the report that caveolin-1 can increase nuclear functions of the phosphorylated AR by binding to and inhibiting the PP1 and PP2A [55].

\section{Clinical implications of AR phosphorylation}

AR phosphorylation has been extensively implicated in pathogeneses, as exemplified by that the development of castrationresistant prostate cancer (CRPC) can be attributed to AR phosphorylation at Tyr267 (by the Ack1 pathway) and Ser515/Ser578 (by the EGFR/MAPK signaling), respectively [56,57]. The enrichment of AR phosphorylation at the NTD indicates one therapeutic strategy is to co-target $\mathrm{AR}$ phosphorylation and ligand binding functions. Indeed, inhibition of phospho-Ser81 can synergize with anti-androgen to disturb CRPC $[36,42]$. Significantly, a recent systematic study based on screening 673 human kinases in PCa cells identified six potential targeting kinases (MAP3K11, DGKD, ICK, CIT, GALK2, and PSKH1), and it is important to assess efficacy of antagonizing these candidates in combination with clinical antiandrogens [58]. In addition, as one frontier in AR research, the receptor phosphorylation has emerged as a potential biomarker in clinical analysis. Indeed, breast and prostate cancer studies have been reported based on immunohistochemistry (IHC) assays with the phospho-Ser213, Ser515, and Ser650 antibodies [31,59-62].These studies can be further substantiated by sufficient antibody validation, such as dose optimization and specific peptide competition analysis.

\section{Conclusions}

In summary, altered and amplified phosphorylation can contribute to abnormal AR activities, including its ligand-independent activation in diseases including PCa. The AR NTD is bestowed with highly selectivity and enriched phosphorylation, providing ample opportunities for specific interventions. Targeting NTD phosphorylation (by kinase and phosphatase modulators) can be applied in synergy with the LBD antagonists (such as antiandrogens) in therapy. Targeting AR phosphorylation is also an option to overcome the AR splicing variants that lose the functional ligand binding capacity and are overexpressed in advanced PCa [63]. Finally, although AR phosphorylation has been extensively studied in receptor activation, its intrinsic connections need to be clarified to AR-mediated transrepression and AR non-genomic functions (like mTOR activation).

\section{Acknowledgements}

This work is supported by the $\mathrm{NIH} / \mathrm{NCI}$ K99/R00 5K99CA135592 (as PI) and NIH P01 CA163227-01A1 (as co-PI) grants to S.Chen. We appreciate the efforts on proofreading by Yan Feng (EdM, Harvard Graduate School of Education).

\section{References}

1. Xu Y, Chen SY, Ross KN, Balk SP (2006) Androgens induce prostate cancer cell proliferation through mammalian target of rapamycin activation and posttranscriptional increases in cyclin D proteins. Cancer Res 66: 7783-7792. 
2. Lin C, Yang L, Tanasa B, Hutt K, Ju BG, et al. (2009) Nuclear receptor-induced chromosomal proximity and DNA breaks underlie specific translocations in cancer. Cell 139: 1069-1083.

3. Haffner MC, Aryee MJ, Toubaji A, Esopi DM, Albadine R, et al. (2010) Androgen-induced TOP2B-mediated double-strand breaks and prostate cancer gene rearrangements. Nat Genet 42: 668-675

4. Cai C, He HH, Chen S, Coleman I, Wang H, et al. (2011) Androgen receptor gene expression in prostate cancer is directly suppressed by the androgen receptor through recruitment of lysine-specific demethylase 1. Cancer Cell 20: 457-471

5. Fang Z, Zhang T, Dizeyi N, Chen S, Wang H, et al. (2012) Androgen Receptor Enhances p27 Degradation in Prostate Cancer Cells through Rapid and Selective TORC2 Activation. J Biol Chem 287: 2090-2098.

6. Zhao JC, Yu J, Runkle C, Wu L, Hu M, et al. (2012) Cooperation between Polycomb and androgen receptor during oncogenic transformation. Genome Res 22: 322-331.

7. Goueli SA, Holtzman JL, Ahmed K (1984) Phosphorylation of the androgen receptor by a nuclear cAMP-independent protein kinase. Biochem Biophys Res Commun 123: 778-784

8. Golsteyn EJ, Goren HJ, Lehoux JG, Lefebvre YA (1990) Phosphorylation and nuclear processing of the androgen receptor. Biochem Biophys Res Commun 171: 336-341.

9. van Laar JH, Bolt-de Vries J, Zegers ND, Trapman J, Brinkmann AO (1990) Androgen receptor heterogeneity and phosphorylation in human LNCaP cells. Biochem Biophys Res Commun 166: 193-200.

10. van Laar JH, Berrevoets CA, Trapman J, Zegers ND, Brinkmann AO (1991) Hormone-dependent androgen receptor phosphorylation is accompanied by receptor transformation in human lymph node carcinoma of the prostate cells. J Biol Chem 266: 3734-3738.

11. Brinkmann AO (2001) Lessons to be learned from the androgen receptor. Eur J Dermatol 11: 301-303.

12. Gioeli D, Ficarro SB, Kwiek JJ, Aaronson D, Hancock M, et al. (2002) Androgen receptor phosphorylation. Regulation and identification of the phosphorylation sites. J Biol Chem 277: 29304-29314.

13. Wong HY, Burghoorn JA, Van Leeuwen M, De Ruiter PE, Schippers E, et al (2004) Phosphorylation of androgen receptor isoforms. Biochem J 383: 267 276.

14. Jenster G, de Ruiter PE, van der Korput HA, Kuiper GG, Trapman J, et al. (1994) Changes in the abundance of androgen receptor isotypes: effects of ligand treatment, glutamine-stretch variation, and mutation of putative phosphorylation sites. Biochemistry 33: 14064-14072.

15. Kuiper GG, de Ruiter PE, Trapman J, Boersma WJ, Grootegoed JA, et al (1993) Localization and hormonal stimulation of phosphorylation sites in the LNCaP-cell androgen receptor. Biochem J 291 : 95-101.

16. Kuiper GG, Brinkmann AO (1995) Phosphotryptic peptide analysis of the human androgen receptor: detection of a hormone-induced phosphopeptide. Biochemistry 34: 1851-1857.

17. Zhou ZX, Kemppainen JA, Wilson EM (1995) Identification of three prolinedirected phosphorylation sites in the human androgen receptor. Mol Endocrinol 9: 605-615.

18. lakoucheva LM, Radivojac P, Brown CJ, O'Connor TR, Sikes JG, et al. (2004) The importance of intrinsic disorder for protein phosphorylation. Nucleic Acids Res 32: 1037-1049.

19. Wang LG, Liu XM, Kreis W, Budman DR (1999) Phosphorylation/ dephosphorylation of androgen receptor as a determinant of androgen agonistic or antagonistic activity. Biochem Biophys Res Commun 259: 21-28.

20. Ueda T, Bruchovsky N, Sadar MD (2002) Activation of the androgen receptor $\mathrm{N}$-terminal domain by interleukin-6 via MAPK and STAT3 signal transduction pathways. J Biol Chem 277: 7076-7085.

21. Sugita S, Kawashima H, Tanaka T, Kurisu T, Sugimura K, et al. (2004) Effect of type I growth factor receptor tyrosine kinase inhibitors on phosphorylation and transactivation activity of the androgen receptor in prostate cancer cells: Ligand-independent activation of the $\mathrm{N}$-terminal domain of the androgen receptor. Oncol Rep 11: 1273-1279.

22. Mahajan NP, Liu Y, Majumder S, Warren MR, Parker CE, et al. (2007) Activated
Cdc42-associated kinase Ack1 promotes prostate cancer progression via androgen receptor tyrosine phosphorylation. Proc Natl Acad Sci U S A 104 8438-8443.

23. Shu SK, Liu Q, Coppola D, Cheng JQ (2010) Phosphorylation and activation of androgen receptor by Aurora-A. J Biol Chem 285: 33045-33053.

24. Lin HK, Wang L, Hu YC, Altuwaijri S, Chang C (2002) Phosphorylationdependent ubiquitylation and degradation of androgen receptor by Akt require Mdm2 E3 ligase. EMBO J 21: 4037-4048.

25. DaSilva J, Gioeli D, Weber MJ, Parsons SJ (2009) The neuroendocrine-derived peptide parathyroid hormone-related protein promotes prostate cancer cell growth by stabilizing the androgen receptor. Cancer Res 69: 7402-7411.

26. Liu T, Li Y, Gu H, Zhu G, Li J, et al. (2013) p21-Activated kinase 6 PAK6 inhibits prostate cancer growth via phosphorylation of androgen receptor and tumorigenic E3 ligase murine double minute-2 Mdm2. J Biol Chem 288: 3359 3369

27. Fu M, Rao M, Wu K, Wang C, Zhang X, et al. (2004) The androgen recepto acetylation site regulates cAMP and AKT but not ERK-induced activity. J Bio Chem 279: 29436-29449

28. Gioeli D, Black BE, Gordon V, Spencer A, Kesler CT, et al. (2006) Stress kinase signaling regulates androgen receptor phosphorylation, transcription, and localization. Mol Endocrinol 20: 503-515.

29. Zong H, Chi Y, Wang Y, Yang Y, Zhang L, et al. (2007) Cyclin D3/CDK11p58 complex is involved in the repression of androgen receptor. Mol Cell Biol 27 : 7125-7142.

30. Chymkowitch P, Le May N, Charneau P, Compe E, Egly JM (2011) The phosphorylation of the androgen receptor by TFIIH directs the ubiquitin proteasome process. EMBO J 30: 468-479.

31. Willder JM, Heng SJ, McCall P, Adams CE, Tannahill C, et al. (2013) Androgen receptor phosphorylation at serine 515 by Cdk 1 predicts biochemical relapse in prostate cancer patients. Br J Cancer 108: 139-148.

32. Funderburk SF, Shatkina L, Mink S, Weis Q, Weg-Remers S, et al. (2009) Specific N-terminal mutations in the human androgen receptor induce cytotoxicity. Neurobiol Aging 30: 1851-1864

33. Choong CS, Kemppainen JA, Wilson EM (1998) Evolution of the primate androgen receptor: a structural basis for disease. J Mol Evol 47: 334-342.

34. Beitel LK, Alvarado C, Mokhtar S, Paliouras M, Trifiro M (2013) Mechanisms mediating spinal and bulbar muscular atrophy: investigations into polyglutamineexpanded androgen receptor function and dysfunction. Front Neurol 4: 53

35. Lieberman AP, Harmison G, Strand AD, Olson JM, Fischbeck KH (2002) Altered transcriptional regulation in cells expressing the expanded polyglutamine androgen receptor. Hum Mol Genet 11: 1967-1976.

36. Chen S, Xu Y, Yuan X, Bubley GJ, Balk SP (2006) Androgen recepto phosphorylation and stabilization in prostate cancer by cyclin-dependen kinase 1. Proc Natl Acad Sci U S A 103: 15969-15974.

37. Gordon V, Bhadel S, Wunderlich W, Zhang J, Ficarro SB, et al. (2010) CDK9 regulates AR promoter selectivity and cell growth through serine 81 phosphorylation. Mol Endocrinol 24: 2267-2280.

38. Hsu FN, Chen MC, Chiang MC, Lin E, Lee YT, et al. (2011) Regulation of androgen receptor and prostate cancer growth by cyclin-dependent kinase 5 . J Biol Chem 286: 33141-33149.

39. Chen S, Gulla S, Cai C, Balk SP (2012) Androgen receptor serine 81 phosphorylation mediates chromatin binding and transcriptional activation. $J$ Biol Chem 287: 8571-8583

40. Kesler CT, Gioeli D, Conaway MR, Weber MJ, Paschal BM (2007) Subcellula localization modulates activation function 1 domain phosphorylation in the androgen receptor. Mol Endocrinol 21: 2071-2084.

41. Liu S, Yuan Y, Okumura Y, Shinkai N, Yamauchi H (2010) Camptothecin disrupts androgen receptor signaling and suppresses prostate cancer cell growth. Biochem Biophys Res Commun 394: 297-302.

42. Hsu FN, Yang MS, Lin E, Tseng CF, Lin H (2011) The significance of Her2 on androgen receptor protein stability in the transition of androgen requirement in prostate cancer cells. Am J Physiol Endocrinol Metab 300: E902-908.

43. Shigemura K, Isotani S, Wang R, Fujisawa M, Gotoh A, et al. (2009) Soluble 
factors derived from stroma activated androgen receptor phosphorylation in human prostate LNCaP cells: roles of ERK/MAP kinase. Prostate 69: 949-955.

44. Sato N, Yoshida M, Ota M (1992) Effects of androgen on phosphorylation of chromosomal proteins in mouse submandibular gland. Biochem Int 26: 843852.

45. Black BE, Vitto MJ, Gioeli D, Spencer A, Afshar N, et al. (2004) Transient, ligand-dependent arrest of the androgen receptor in subnuclear foci alters phosphorylation and coactivator interactions. Mol Endocrinol 18: 834-850.

46. La Montagna R, Caligiuri I, Maranta P, Lucchetti C, Esposito L, et al. (2012) Androgen receptor serine 81 mediates Pin 1 interaction and activity. Cell Cycle 11: $3415-3420$

47. Solit DB, Scher HI, Rosen N (2003) Hsp90 as a therapeutic target in prostate cancer. Semin Oncol 30: 709-716.

48. Haelens A, Tanner T, Denayer S, Callewaert L, Claessens F (2007) The hinge region regulates DNA binding, nuclear translocation, and transactivation of the androgen receptor. Cancer Res 67: 4514-4523.

49. Clinckemalie L, Vanderschueren D, Boonen S, Claessens F (2012) The hinge region in androgen receptor control. Mol Cell Endocrinol 358: 1-8.

50. Blok LJ, de Ruiter PE, Brinkmann AO (1998) Forskolin-induced dephosphorylation of the androgen receptor impairs ligand binding. Biochemistry 37: 3850-3857.

51. Yang CS, Vitto MJ, Busby SA, Garcia BA, Kesler CT, et al. (2005) Simian virus 40 small $t$ antigen mediates conformation-dependent transfer of protein phosphatase 2A onto the androgen receptor. Mol Cell Biol 25: 1298-1308.

52. Bhardwaj A, Singh S, Srivastava SK, Honkanen RE, Reed E, et al. (2011) Modulation of protein phosphatase $2 \mathrm{~A}$ activity alters androgen-independent growth of prostate cancer cells: therapeutic implications. Mol Cancer Ther 10 720-731.

53. Bluemn EG, Spencer ES, Mecham B, Gordon RR, Coleman I, et al. (2013) PPP2R2C loss promotes castration-resistance and is associated with increased prostate cancer-specific mortality. Mol Cancer Res 11: 568-578.
54. Chen S, Kesler CT, Paschal BM, Balk SP (2009) Androgen receptor phosphorylation and activity are regulated by an association with protein phosphatase 1. J Biol Chem 284: 25576-25584.

55. Li L, Ren CH, Tahir SA, Ren C, Thompson TC (2003) Caveolin-1 maintains activated Akt in prostate cancer cells through scaffolding domain binding site interactions with and inhibition of serine/threonine protein phosphatases PP and PP2A. Mol Cell Biol 23: 9389-9404.

56. Ponguta LA, Gregory CW, French FS, Wilson EM (2008) Site-specific androgen receptor serine phosphorylation linked to epidermal growth factor-dependent growth of castration-recurrent prostate cancer. J Biol Chem 283: 20989-21001.

57. Mahajan K, Coppola D, Rawal B, Chen YA, Lawrence HR, et al. (2012) Ack1 mediated androgen receptor phosphorylation modulates radiation resistance in castration-resistant prostate cancer. J Biol Chem 287: 22112-22122.

58. Whitworth H, Bhadel S, Ivey M, Conaway M, Spencer A, et al. (2012) Identification of kinases regulating prostate cancer cell growth using an RNA phenotypic screen. PLoS One 7: e38950.

59. McCall P, Gemmell LK, Mukherjee R, Bartlett JM, Edwards J (2008) Phosphorylation of the androgen receptor is associated with reduced survival in hormone-refractory prostate cancer patients. Br J Cancer 98: 1094-1101.

60. Ha S, Iqbal NJ, Mita P, Ruoff R, Gerald WL, et al. (2012) Phosphorylation of the androgen receptor by PIM1 in hormone refractory prostate cancer. Oncogene

61. Ren Q, Zhang L, Ruoff R, Ha S, Wang J, et al. (2013) Expression of androgen receptor and its phosphorylated forms in breast cancer progression. Cancer 119: $2532-2540$

62. Zellweger T, Stürm S, Rey S, Zlobec I, Gsponer JR, et al.(2013) Estrogen receptor beta expression and androgen receptor phosphorylation correlate with a poor clinical outcome in hormone-naive prostate cancer and are elevated in castration-resistant disease. Endocr Relat Cancer 20: 403-413.

63. Dehm SM, Tindall DJ (2011) Alternatively spliced androgen receptor variants Endocr Relat Cancer 18: R183-196. 